\title{
Utilization of antenatal care services among teenagers in Ethiopia: A cross sectional study
}

\author{
Tewodros Alemayehu, Jemal Haidar, Dereje Habte
}

\begin{abstract}
Background: Teenaged women suffer from a disproportionate share of reproductive health problem. The purpose of this study was to estimate the utilization of antenatal care (ANC) services among teenagers (13-19 years) during delivery in Ethiopia.

Methodology: Raw data collected from all part of the country on child bearing aged women using stratified cluster sampling method by the Ethiopian Demographic Health Survey 2005 was used. From the large dataset of women, a total of 994 teenage women at the time of their most recent childbirth five years prior to the survey was selected and analyzed. Both bivariate and multivariate analyses were performed to determine the differentials of ANC by explanatory variables.

Result: Most (60\%) of the subjects were in the age group between 18 and 19 years. The vast majority (90\%) was from the rural settings and most $(87 \%)$ were in marital union. Almost three out of four $(72.4 \%)$ of those who had given birth has no any form of formal education. Over a quarter (27.3\%) of most recent childbirths had at least one ANC service, of this, $21 \%$ had started their first antenatal visit in the first trimester of pregnancy. The majority $(80.4 \%)$ of the women who attended ANC delivered at home without being assisted. The major deriving factors for the utilization of ANC service were education level of women and their male partners, better wealth index and urban residence.

Conclusion: Education of partners, rich wealth index and urban residence seemed to encourage teenagers to utilize ANC. Appropriate interventions targeting teenaged women with poor socioeconomic status is recommended with more emphasis on the rural underserved segment of population. [Ethiop. J. Health Dev. 2010;24(3):221-225]
\end{abstract}

\section{Introduction}

Adolescence is the transitional period from childhood to adulthood characterized by significant physiological, psychological and social changes. World Health Organization (WHO) defines the age group 10-19 and 15-24 years of age as adolescent and youth, respectively. Those in the age group 10-24 years are called young people (1).

For all women of reproductive age, especially for pregnant women, utilization of health care services is a key proximate determinant of maternal and infant outcomes, including maternal and infant mortality. The benefits of health care seeking are tremendous particularly in settings and subgroups where the socioeconomic and public health resources are constrained. It is evident that timely antenatal care (ANC) is an opportunity to prevent the direct causes of maternal mortalities and reduction of fetal and neonatal deaths related to obstetric complications. Thus, antenatall care is one of the recommended cares to be provided for pregnant women (2).

According to the recent WHO recommendation (although not implemented at the time of Ethiopian Demographic Health Survey, 2005) ANC should be started in the first trimester of pregnancy or early in the second trimester. If the pregnant woman has no serious health problem and does not need special attention, only four ANC visits suffice $(3,4)$. ANC can improve certain outcomes of pregnancy complications such as eclampsia, anemia and syphilis through early detection, management and timely referral of high risk pregnancies, though such care has not been shown to reduce the rates of maternal mortality (4).

Generally, ANC during pregnancy can provide an entry into the health system, and for teenagers in particular such care may be one of the first comprehensive health assessments deemed necessary $(2,4)$. Most importantly, utilization of ANC provides the opportunity to teach teenaged women on how to recognize and respond to the signs of obstetric complications as they may have little knowledge and experience in reproductive health (2). The other added values obtained from ANC services are provision of tetanus toxiod immunization which is lifesaving both for the mother and infant; treatment of malaria, anemia and STIs; and an entry point for prevention of mother to child transmission of HIV. Provision of health advices on birth spacing and use of institutional delivery which would significantly improve both maternal and fetal outcomes are the other important services obtained in the process $(2,4,5)$.

In Ethiopia, the proportion of pregnant women that uses ANC from health professionals is generally very low. In Gondar, 45.7\% of women aged 15-49 years attended $\mathrm{ANC}$ at least once for their most recent birth and those who had got ANC from health professionals were more likely to seek health workers assisted delivery than those 
who didn't get ANC (6). Another similar study in Southern Ethiopia documented $26 \%$ of the women attended ANC for their most recent birth within six years in southern Ethiopia (7) and the national ANC attendees rate for most recent birth in five years was only $27.6 \%$ by the year 2005 (9).

Adolescents are at increased risk of experiencing complication during pregnancy and childbirth and at the same time less likely to use maternal health care services including ANC and delivery from skilled health workers $(2,6,9)$. A range of factors have been attributed to the low utilization of ANC service. Among these, the most commonly mentioned variables are: socio-economic and demographic differences such as educational status, living in rural area, marital status and being in poorest wealth index and obstetric factors such as parity, outcome of previous pregnancy and whether the pregnancy is planned or not $(4,10-13)$.

Against this background information, this work was proposed to identify the factors associated with the utilization of ANC services in the Ethiopian context among teenaged pregnancies and provides evidence based information for the concerned bodies.

\section{Methods}

This study is based on the second largest data set collected by the Ethiopian Demographic and Health Survey (EDHS) 2005 on women of reproductive age in nine regions and two city administrative councils of Ethiopia, namely: Tigray; Afar; Amhara; Oromiya; Somali; Benishangul-Gumuz; Southern Nations, Nationalities and Peoples (SNNP); Gambela; Harari Regions; Addis Ababa and Dire Dawa City Administrations. The EDHS 2005 was conducted from April 29 to August 30, 2005 to provide various socioeconomic, health, nutrition and other related information at country level in a representative sample using stratified cluster sampling method. All the EDHS data in the country were collected by properly trained data collectors using standardized, structured and pre-tested questionnaire. The detail of the EDHS methodology is mentioned elsewhere (9). Although the data appears to be 5-years old and some of the new initiatives like health extension programs were not in place at the time of the study, the information captured during the EDHS-2005 would still reflect important information and help for some improvements if the impeding factors are revealed.

Prior to the analysis, permission was obtained from ORC Macro/CSA and the analysis was performed based on the extracted dataset of teenaged women who had given birth before the age of 20 during the last five years prior to the time of interview. When woman has given more than one birth in the given period, only the most recent birth has been considered for the analysis.

Since the sampling procedure didn't yield equal probability of selection of the samples, weighing of the samples was applied to produce the national estimate to the descriptive part of this article whereas for the logistic regression analysis, weighing of the sample was not used. Data were analyzed using SPSS version 14.

To estimate the factors influencing the practice of ANC utilization among the teenaged women, a range of maternal characters and other important factors were compared between ANC users and their counterpart. Initially, bivariate analysis was performed to determine the differentials of ANC by explanatory variables and then the variables were further tested with multivariate logistic regression models using enter method. Pearson's chi-square test of independence was performed to test the existence of significant association of the important factors with ANC. A p-value of less than 0.05 denoted significance in differences.

The following standard case definitions were applied;

- ANC service utilization refers to skilled antenatal care rendered by the health workers in health institution which is consistent with WHO definition (4).

- Wealth ranking was grossly categorized into 5 major quintiles based on household assets as lowest (poorest), second (poorer), middle (middle), fourth (richer) and highest (richest),

\section{Results}

A total of 994 women aged between 14 and 19 years were extracted from the large data set of women by the time of delivery. Those women were participants that have given birth at least once in the last five years. Most $(60 \%)$ of the subjects were in the age group between 18 and 19 years. The vast majority $(90 \%)$ was from the rural settings and most (89\%) were married. Over three quarter $(99.9 \%)$ of them had no job and almost three out of four $(92.4 \%)$ of those who had given birth has no formal education. Nearly half $(49.3 \%)$ belong to second and middle wealth index categories (Table 1).

Among the 994 women who had given birth before the age of 20 years in the last five years, only $29.3 \%$ had ANC by health workers and $1.3 \%$ were seen by traditional birth attendants and community health agents. About three quarters (71.5\%) did not attend Antenatal ANC during their recent pregnancy. When the frequency of ANC attended by health workers was categorized by trimester, only $21.8 \%$ had sought first ANC visit in the first trimester. Those who started their ANC visit during their second and third trimester were $49.6 \%$ and $30.6 \%$ respectively. Majority (53.4\%) of the teenage mothers had less than four antenatal contacts and the rest 126 $(46.5 \%)$ reported to have four or more visits. Surprisingly, most (80.4\%) of those women who attended ANC delivered at home without assistance from skilled birth attendants (Table 2). 
Table 1: Socio-demographic character of teenager women who had given live birth in five year preceding the survey, Ethiopia, 2005

\begin{tabular}{|c|c|c|}
\hline Variables & Number & Percents \\
\hline \multicolumn{3}{|l|}{ Age at birth (yrs) } \\
\hline $13-15$ & 10 & 1.0 \\
\hline $15-17$ & 384 & 38.6 \\
\hline 18-19 & 600 & 60.4 \\
\hline \multicolumn{3}{|l|}{ Religion } \\
\hline Orthodox & 472 & 47.5 \\
\hline Muslim & 344 & 34.6 \\
\hline Protestant & 145 & 14.6 \\
\hline Others & 33 & 3.3 \\
\hline \multicolumn{3}{|l|}{ Setting } \\
\hline Urban & 99 & 10.0 \\
\hline Rural & 895 & 90.0 \\
\hline \multicolumn{3}{|l|}{ Marital status } \\
\hline Never married & 15 & 1.5 \\
\hline Married & 865 & 87.0 \\
\hline Living together & 20 & 2.0 \\
\hline Widowed & 8 & 0.8 \\
\hline Divorced & 63 & 6.4 \\
\hline Not living together & 23 & 2.3 \\
\hline \multicolumn{3}{|l|}{ Education } \\
\hline No education & 720 & 72.4 \\
\hline Primary & 235 & 23.6 \\
\hline Secondary & 39 & 3.9 \\
\hline \multicolumn{3}{|l|}{ Wealth index } \\
\hline Lowest & 177 & 17.8 \\
\hline Second & 234 & 23.5 \\
\hline Middle & 247 & 23.8 \\
\hline Fourth & 175 & 17.6 \\
\hline Highest & 171 & 17.2 \\
\hline \multicolumn{3}{|l|}{ Occupation } \\
\hline Not working & 772 & 77.7 \\
\hline Working & 222 & 22.3 \\
\hline
\end{tabular}

The various factors associated with the utilization of ANC services are shown in Table 3. In the bivariate analysis; religion, women and partner educations, place of residence, gender of the household-head, wealth index and subjects listening to radio or watching television at least once in a week were significantly associated with utilization of the ANC service. Whereas age, marital status, occupation and obstetric factors like parity, history of death of children and the child birth being planned were not associated with the utilization of the service. Nevertheless, in the multivariate analysis, only four factors retained their significant associations with the use of the ANC services. These were education of the teenaged women, educational level of male partners, rich wealth index and urban residence after controlling for age at birth, religion, marital status, occupation, listening radio or watch television at least once in a week, gender of household head, obstetric history ranging from history of death of child, parity and child birth being planned or not. Teenaged who had primary and secondary educational level were $1.5(\mathrm{AOR}=1.5$ and $95 \% \mathrm{CI}=$ $1.04,2.3)$ and four times $(\mathrm{AOR}=4.1$ and $95 \% \mathrm{CI}=1.9$, 8.5) more likely to use ANC service respectively than those who had no education. Similarly, women's partner education level was also found to be the other predictor implying that those whose partner had secondary or more education level were 2.5 times more likely to seek ANC than those whose partner has no education. Teenagers belonging to the highest $(\mathrm{AOR}=3.5$ and $95 \% \mathrm{CI}=1.9$, $6.5)$, the fourth $(\mathrm{AOR}=2.4$ and $95 \% \mathrm{CI}=1.5,4.2)$, middle $(\mathrm{AOR}=1.8$ and $95 \% \mathrm{CI}=1.1,3.1)$ and second $(\mathrm{AOR}=2.0$ and $95 \% \mathrm{CI}=1.2 .3 .4)$ wealth quintile were more likely to use ANC than those belonging to the lowest wealth quintile after controlling for potential confounders. Likewise, those teenaged women living in urban settings were two times more likely to seek ANC services than their counterparts (AOR $=1.9$ and $95 \% \mathrm{CI}$ $=1.1,3.4)$.

\begin{tabular}{|c|c|c|}
\hline Variables & Number & Percents \\
\hline \multicolumn{3}{|l|}{ Source of ANC (n=994) } \\
\hline Health workers & 271 & 27.3 \\
\hline Trained traditional birth attendants & 6 & 0.6 \\
\hline Untrained traditional birth attendants & 2 & 0.1 \\
\hline Community health workers and others & 5 & 0.5 \\
\hline No one & 710 & 71.5 \\
\hline \multicolumn{3}{|l|}{ Timing of first ANC $(n=271)$} \\
\hline $1^{\mathrm{st}}$ trimester & 59 & 21.8 \\
\hline $2^{\text {nd }}$ trimester & 129 & 47.6 \\
\hline $3^{\text {rd }}$ trimester & 81 & 29.9 \\
\hline \multicolumn{3}{|l|}{ Number of ANC visits $(n=271)$} \\
\hline Less than four & 145 & 53.5 \\
\hline Four or more & 126 & 46.5 \\
\hline \multicolumn{3}{|l|}{ Institutional deliveries $(n=271)$} \\
\hline Yes & 53 & 19.6 \\
\hline No & 218 & 80.4 \\
\hline
\end{tabular}




\section{Discussion}

As in the case of general population, the rate of ANC utilization was low among teenager women in Ethiopia. Only $29.3 \%$ of the teenagers have used ANC service rendered by health workers for their most recent birth in five years prior to this study. Such a low rate of use was also documented in the country $(6,14)$. However when compared with other neighboring countries, the rate observed in Ethiopia is lower. For instance, the rate of ANC utilization among teenaged women at most recent birth in five years was $92 \%, 84 \% 89 \%, 93 \%, 94 \%$ and 95\% in Eritrea, Kenya, Mozambique, Rwanda, Malawi, Tanzania and Uganda, respectively (15).

The reasons attributed for such a low utilization in most of the above mentioned countries were inadequate information about the existence of the service, ignorance, fear, shyness, distance of obtaining the service, culture, economic reason, and un-friendly approach of the health workers $(3,16)$. The fact that the large majority of the study participants were from rural setting presumably with little access to information and health services could be the reason behind lower ANC utilization observed in our study during the study period. Nonetheless, this assertion might have been different if the study is conducted presently as there is currently a new innovative health information packages through the health extension program. The health extension program consist of a range of health related information that includes reproductive health information.

Another important finding observed in the study is the impact of education on utilization of ANC. The findings of this study is consistent with previous studies reported elsewhere in the world including Ethiopia $(6,10,13-14$, 17). Education is likely to enhance female autonomy so that women develop greater confidence and capability to make decisions for their own health. It is also likely that educated women seek higher quality services and have greater ability to use health care inputs that offer better care (14).

In this study teenagers living in household that fall within the poorest population quintile used ANC services less frequently than those in the richest $20 \%$ category. In addition, this study has shown some degree of wealth differential to exist, as is the case everywhere - the gap between the richest $20 \%$ and the poorest $20 \%$ for use of antenatal care varies enormously. Our finding is comparable with some previous studies, which documented the impact of economic status towards the use of ANC provided by health professionals $(6,10,13$, 17).

Similarly, teenagers living in urban areas were two times more likely to use ANC than those living in rural areas after controlling for potential socioeconomic, demographic and obstetric variables, again showing comparable results with the aforementioned studies (14, 17). This may be because urban dwellers may have better access to health facilities as well as to health related information (14).

In conclusion, the utilization of ANC service among teenaged pregnant women was low in the country; secondly, most of them were found to get the service late either in second or third trimesters. The major determinant factors for utilizing ANC were having formal education of both female and male partner, living in urban settings and rich wealth index category. Appropriate interventions targeting teenagers with poor socioeconomic status is recommended with emphasis on the rural underserved population.

\section{Acknowledgements}

We would like to acknowledge the owner of the raw data, ORC macro/CSA international, for their permission to use the data. We would also like to appreciate school of public health of Addis Ababa University for their financial support. This paper is part of a thesis submitted for degree of Master of Public Health.

\section{References}

1. WHO. Programming for teenaged health and development report of WHO/UNFPA/UNICEF study group on programming for adolescents health. Geneva: WHO; 1999.

2. Reynolds H.W, Wong E.L, Tucker H. Adolescents' use of Maternal and Child Health Services in Developing Countires. International Family Planning Perspectives 2006 March;23(1):6-16.

3. Bearinger L.H, Sieving R.E, Ferguson J, Sharma V. Global perspectives on the sexual and reproductive health of adolescents; patterns, prevention, and potential. Lancet 2007;369:1220-31.

4. WHO/UNICEF. Antenatal care in developing countries: promises, achievements and missed opportunities: an analysis of trends, levels and differentials, 1990-2001. Geneva: WHO; 2003.

5. Stoltzfus R.J. Iron-Deficiency Anemia: Reexamining the Nature and Magnitude of the Public Health Problem. Journal of Nutrition 2001;131:697S-701S.

6. Nigusie M, Haile Mariam D, Mitke G. Assessment of safe delivery service utilization among women of child bearing age in North Gondar, Northwest Ethiopia. Ethiop. J. Health Dev 2004;18(3):145-52.

7. Mekonnen Y. Patterns of maternity care service utilization in Southern Ethiopia: Evidence from a community and family survey. Ethiop. J. Health Dev 2003;17(1):27-33.

8. CSA, ORC Macro. Ethiopia Demography and Health Survey 2005. Addis Ababa, Ethiopia and Calverton, Maryland, USA: Central statistical agency and ORC Macro: Addis Ababa; 2006.

9. Watcharaseranee N, Pinchantra P, Piyaman S. The Incidence and Complications of Teenage Pregnancy at Chonburi Hospital. Journal of Medical Association Thailand 2006;89(4):118-23.

10. Fenta M. Assessment of factors affecting utilization of maternal health care services in Assaita and Dubti 
towns, Afar Regional State, North East Ethiopia. MSc. Thesis, Addis Ababa: Addis Ababa University; 2005.

11. Eloundou Enyegue PM, Stokes CS. Teen Fertility and Gender Inequality in Education: A Contextual Hypothesis. Demographic Research 2004 December 08;11(11):305-34.

12. Biratu BT, Lindsrom DP. The influence of husband approval on women's use of antenatal care; result from Yirgalem and Jimma towns, southwest Ethiopia. Ethiop. J. Health Dev 2000;20(2):84-92.

13. Fantahun M. Olwit G and Shamabo D Determinants of antenatal care attendance and preference of site of delivery in Addis Ababa. Ethiop. J. Health Dev 1992;6(2):17-21.
14. Mekonnen Y. Utilization of maternal health care services in Ethiopia. Calverton, Maryland, USA: ORC Macro; 2002.

15. Macro International INC. measure DHS STAT compiler. 2008 (cited May 10, 2008); Available from: URL: http://www.measuredhs.com

16. Seifu A. Reproductive health needs of urban and rural out of school adolescents in East Gojjam: A cross sectional comparative study. [Masters thesis]: Addis Ababa University; 2001.

17. Woldemichael G. Teenage Childbearing and Child Health in Eritrea: Max Planck Institute for Demographic Research; 2005. 